Pacific

Journal of

Mathematics

LE SYSTÈME DIFFÉRENTIEL DE HÉNON-HEILES ET LES VARIÉTÉS DE PRYM

A. LESFARI

Volume $212 \quad$ No. 1

November 2003 


\title{
LE SYSTÈME DIFFÉRENTIEL DE HÉNON-HEILES ET LES VARIÉTÉS DE PRYM
}

\author{
A. LESFARI
}

On montre que la fibre $\mathbf{F}$ définie par l'intersection des invariants du système différentiel de Hénon-Heiles se complète en une surface abélienne $\widetilde{\mathbf{F}}$, par l'adjonction d'une surface de Riemann $\Gamma$ lisse hyperelliptique de genre 3 ; laquelle est un revêtement double ramifié le long d'une courbe elliptique $\Gamma_{0}$. Aussi $\widetilde{\mathbf{F}}$ peut être identifiée à la duale d'une variété de Prym $\operatorname{Prym}\left(\Gamma / \Gamma_{0}\right)$ et le système se linéarise sur cette variété.

\section{Position du problème.}

Le système différentiel de Hénon-Heiles [7] s'écrit sous la forme

$$
\left\{\begin{array}{l}
\dot{q}_{1}=p_{1}, \\
\dot{q}_{2}=p_{2}, \\
\dot{p}_{1}=-A q_{1}-2 q_{1} q_{2}, \\
\dot{p}_{2}=-B q_{2}-q_{1}^{2}-\varepsilon q_{2}^{2},
\end{array}\right.
$$

où $\mathrm{A}, \mathrm{B}, \varepsilon$ sont des constantes et admet les invariants (intégrales premières) suivants:

(i) $\operatorname{Pour} \varepsilon=1$, on a

$$
\begin{aligned}
& H_{1}=\frac{1}{2}\left(p_{1}^{2}+p_{2}^{2}\right)+q_{1}^{2} q_{2}+\frac{1}{3} q_{2}^{3}, \\
& H_{2}=p_{1} p_{2}+\frac{1}{3} q_{1}^{3}+q_{1} q_{2}^{2} .
\end{aligned}
$$

(ii) Pour $\varepsilon=6$, on a

$$
\begin{aligned}
& H_{1}=\frac{1}{2}\left(p_{1}^{2}+p_{2}^{2}+A q_{1}^{2}+B q_{2}^{2}\right)+q_{1}^{2} q_{2}+6 q_{2}^{3}, \\
& H_{2}=q_{1}^{4}+4 q_{1}^{2} q_{2}^{2}-4 p_{1}\left(p_{1} q_{2}-p_{2} q_{1}\right)+4 A q_{1}^{2} q_{2}+(4 A-B)\left(p_{1}^{2}+A q_{1}^{2}\right) .
\end{aligned}
$$

L'intégration des équations (1.1) dans le cas $\varepsilon=1$, s'effectue au moyen d'intégrales elliptiques et ne pose pas de problèmes. Le cas $\varepsilon=6$, est plus intéressant mais plus compliqué [4] et [5]. Lorsque $A=B=0$, Adler et van Moerbeke [2] ont montré que ce cas est lié par une transformation 
birationnelle au problème de Kowalewski ainsi qu'au flot géodésique sur $S O$ (4) pour une métrique de Manakov. Le but de cette note est d'étudier géométriquement et d'une manière rigoureuse ce problème pour $A$ et $B$ quelconque. Dans tout ce qui va suivre, on pose $\varepsilon=6$.

\section{Complète intégrabilité algébrique.}

Considérons un systéme hamiltonian complètement intégrable

$$
X_{H}: \quad \dot{x}=J \frac{\partial H}{\partial x}, \quad x \in \mathbb{R}^{2 n}, J=\left(\begin{array}{ll}
0 & -I \\
I & 0
\end{array}\right),
$$

où $H$ est l'Hamiltonian et $I$ est la matrice unité. Le système (2.1) possède $n$ intégrales premières $H_{1}=H, H_{2}, \ldots, H_{n}$ en involution et indépendantes. Pour presque tous les $c_{i} \in \mathbb{R}$, les variétés invariantes

$$
\bigcap_{i=1}^{n}\left\{x \in \mathbb{R}^{2 n}: H_{i}(x)=c_{i}\right\},
$$

sont compactes, connexes et par le théorème d'Arnold-Liouville [3] et [18], elles sont difféomorphes aux tores réels $\mathbb{R}^{n} /$ réseau sur lesquels les flots $g_{i}^{t}(x)$ définies par les champs de vecteurs $X_{H_{i}}, 1 \leq i \leq n$, sont des mouvements rectilignes.

Soient $x \in \mathbb{C}^{2 n}, t \in \mathbb{C}$ et $\Delta \subset \mathbb{C}^{2 n}$ un ouvert de Zariski. Notons que l'application moment

$$
\varphi:\left(H_{1}, \ldots, H_{n}\right): \mathbb{C}^{2 n} \rightarrow \mathbb{C}^{n},
$$

est submersive sur $\Delta$. Soit

$$
\begin{aligned}
\Pi & =\varphi\left(\mathbb{C}^{2 n} \backslash \Delta\right), \\
& =\left\{c=\left(c_{i}\right) \in \mathbb{C}^{n}: \exists x \in \varphi^{-1}(c) \text { avec } d H_{1}(x) \wedge \cdots \wedge d H_{n}(x)=0\right\},
\end{aligned}
$$

le lieu critique de $\varphi$ où $c=\left(c_{i}\right)$ est le point courant de $\mathbb{C}^{2 n}$ et soit $\bar{\Pi}$ la fermeture de Zariski dans $\mathbb{C}^{4}$. Rappelons [1] et [14] que le système (2.1) est algébriquement complètement intégrable si pour $c \in \mathbb{C}^{n} \backslash \bar{\Pi}$, la fibre $\mathbf{F}=\varphi^{-1}(c)$ est la partie affine d'une variété abélienne (tore complexe algébrique $\widetilde{\mathbf{F}} \simeq \mathbb{C}^{n} /$ réseau), les flots $g_{i}^{t}(x), x \in \mathbf{F}, t \in \mathbb{C}$, définies par les champs de vecteurs $X_{H_{i}}, 1 \leq i \leq n$, sont des mouvements rectilignes sur $\widetilde{\mathbf{F}}$ et les coordonnées $x_{i}=x_{i}\left(t_{1}, \ldots, t_{n}\right)$ du problème sont des fonctions méromorphes de $\left(t_{1}, \ldots, t_{n}\right)$. En outre, si le flot hamiltonian (2.1) est algébriquement complètement intégrable, alors ce système admet des solutions sous la forme de séries de Laurent en $t$ telles que chaque $x_{i}$ explose pour au moins une valeur finie de $t$ et les séries de Laurent de $x_{i}$ admettent $n-1$ paramètres libres. 
Le système (1.1) s'écrit sous la forme (2.1) avec $n=2$. Plus précisément, on a

$$
\stackrel{\bullet}{x} \equiv f(x)=J \frac{\partial H}{\partial x},
$$

avec $x=\left(q_{1}, q_{2}, p_{1}, p_{2}\right)$ et $H=H_{1}$ (1.2). Comme l'application polynomiale est continue pour la topologie de Zariski, l'ensemble

$$
\left\{x \in \mathbb{C}^{4}: \varphi(x) \in \mathbb{C}^{4} \backslash \bar{\Pi}\right\},
$$

est un ouvert de Zariski dans $\mathbb{C}^{4}$. On cherche à montrer que pour $c \in \mathbb{C}^{4} \backslash$ $\bar{\Pi}$, la fibre

$$
\begin{aligned}
\mathbf{F} & =\varphi^{-1}(c), \\
& =\bigcap_{i=1}^{2}\left\{x \in \mathbb{C}^{4}: H_{i}(x)=c_{i}\right\},
\end{aligned}
$$

forme la partie affine d'une surface abélienne et qu'en outre les flots définis par les champs de vecteurs hamiltoniens (engendrés par $H_{1}$ et $H_{2}$ ) sont des mouvements rectilignes sur cette surface abélienne. On procède comme suit: d'abord l'on montre l'existence de solutions $x=\left(q_{1}, q_{2}, p_{1}, p_{2}\right)$ du système (2.2) sous la forme de séries de Laurent

$$
\begin{cases}q_{1}=\frac{q_{1}^{(0)}}{t}+q_{1}^{(1)}+q_{1}^{(2)} t+q_{1}^{(3)} t^{2}+\ldots, & p_{1}=\dot{q}_{1}, \\ q_{2}=\frac{q_{2}^{(0)}}{t^{2}}+\frac{q_{2}^{(1)}}{t}+q_{2}^{(2)}+q_{2}^{(3)} t+q_{2}^{(4)} t^{2}+\ldots, & p_{2}=\dot{q}_{2},\end{cases}
$$

dépendant de trois paramètres libres: $\alpha, \beta, \gamma$. En substituant ces développements dans le système $(2.2)$, on voit que les coefficients $x^{(0)}, x^{(1)}, \ldots$, satisfont aux équations

$$
x^{(0)}+f\left(x^{(0)}\right)=0,
$$

$$
(L-k I) x^{(k)}=\text { polynôme en } x^{(0)}, x^{(1)}, \ldots, x^{(k-1)}, k \geq 1,
$$

où $L$ est la matrice jacobienne de (2.5). Les trois paramètres libres $\alpha, \beta$ et $\gamma$ apparaissent respectivement dans l'équation (2.5), l'équation (2.6) pour $k=1$ et l'équation (2.6) pour $k=6$. L'étape suivante est fondamentale et consiste à considérer l'ensemble

$\Gamma=$ fermeture des composantes continues de \{séries de Laurent de $x(t)$ tels que: $H_{1}(x)=c_{1}$ et $\left.H_{2}(x)=c_{2}\right\}$, $=\bigcap_{i=1}^{2}\left\{\right.$ coefficient de $t^{0}$ dans $\left.H_{i}(x(t))=c_{i}\right\}$, $=$ deux relations polynomiales entre les variables $\alpha, \beta$ et $\gamma$, $=$ une surface de Riemann hyperelliptique de genre 3 d'équation: 


$$
a_{1} \beta^{2}+a_{2} \alpha^{8}+a_{3} \alpha^{6}+a_{4} \alpha^{4}+a_{5} \alpha^{2}+a_{6}=0
$$

où

$$
\begin{gathered}
a_{1}=36, \quad a_{2}=\frac{7}{432}, \quad a_{3}=\frac{5}{12} A-\frac{13}{216} B, \\
a_{4}=\frac{671}{15120} B^{2}+\frac{17}{7} A^{2}-\frac{943}{1260} B A, \\
a_{5}=\frac{2}{9} A B^{2}-\frac{1}{2520} B^{3}-\frac{10}{7} c_{1}-\frac{13}{6} A^{2} B+4 A^{3}, \quad a_{6}=-c_{2} .
\end{gathered}
$$

Notons que l'application

$$
\sigma: \Gamma \rightarrow \Gamma,(\alpha, \beta) \mapsto(-\alpha, \beta),
$$

est une involution sur $\Gamma$ et que cette dernière est un revêtement double

$$
\Gamma \rightarrow \Gamma_{0},(\alpha, \beta) \mapsto(\zeta, \beta),
$$

ramifié en 4 points d'une courbe elliptique:

$$
\Gamma_{0}: a_{1} \beta^{2}+a_{2} \zeta^{4}+a_{3} \zeta^{3}+a_{4} \zeta^{2}+a_{5} \zeta+a_{6}=0 .
$$

Par conséquent, on a le:

Théorème 1. Le système d'équations différentielles (2.2) admet une famille de solutions en séries de Laurent méromorphes (2.4) dépendant de trois paramètres libres. En outre, le diviseur $\Gamma$ (2.7) des poles des fonctions $x=\left(q_{1}, q_{2}, p_{1}, p_{2}\right)$ est une surface de Riemann lisse hyperelliptique de genre 3 ; c'est un revêtement double ramifié en quatre points d'une courbe elliptique $\Gamma_{0}(2.10)$.

On va procèder maintenant à la compactification de la fibre $\mathbf{F}$ (2.3) en une surface abélienne $\widetilde{\mathbf{F}}$. La méthode consiste à plonger $\mathbf{F}$ dans l'espace projectif complexe $\mathbb{P}^{7}(\mathbb{C})$ à l'aide des fonctions de $\mathbb{L}(2 \Gamma)$. Ce sont des fonctions polynomiales $\left(1, f_{1}, \ldots, f_{7}\right)$ ayant au pire un pôle double de telle façon que:

$$
\operatorname{dim} \mathbb{L}(2 \Gamma)=\text { genre de }(2 \Gamma)-1=8 .
$$

Par ailleurs, on montre qu'il existe sur la surface $\widetilde{\mathbf{F}}$ deux différentielles holomorphes $d t_{1}$ et $d t_{2}$ telles que:

$$
\left.d t_{1}\right|_{\Gamma}=\omega_{1},\left.d t_{2}\right|_{\Gamma}=\omega_{2},
$$

où $\omega_{1}, \omega_{2}$ sont des différentielles holomorphes (voir Section 3, pour une expression explicite) sur la surface de Riemann $\Gamma$. En outre, l'espace des différentielles holomorphes sur $\Gamma$ est

$$
\left\{f_{i}^{(0)} \omega_{2}, 1 \leq i \leq 7\right\} \oplus\left\{\omega_{1}, \omega_{2}\right\}
$$


où les $f_{i}^{(0)}$ sont les premiers coefficients des fonctions $f_{i} \in \mathbb{L}(2 \Gamma)$ et le plongement de $\Gamma$ dans $\mathbb{P}^{7}(\mathbb{C})$ est à deux différentielles holomorphes prés le plongement canonique:

$$
(\alpha, \beta) \in \Gamma \mapsto\left[\omega_{2}, f_{1}^{(0)} \omega_{2}, \ldots, f_{7}^{(0)} \omega_{2}\right] \in \mathbb{P}^{7}(\mathbb{C}) .
$$

La suite consiste à montrer que les orbites du champ de vecteurs (2.2) passant à travers $\Gamma$ forment une surface lisse $S$ tout le long de $\Gamma$ tel que: $S \backslash$ $\Gamma \subseteq \mathbf{F}$. Alors, on prouve que $\widetilde{\mathbf{F}}=\mathbf{F} \cup S$ est une variété compacte (grâce au fait que les solutions issues des points de $\Gamma$ pénètrent immédiatement dans la partie affine $\mathbf{F}$, plongée dans $\mathbb{P}^{7}(\mathbb{C})$ à l'aide des fonctions de $\mathbb{L}(2 \Gamma)$ ) et est munie de deux champs de vecteurs réguliers, indépendants en chaque point et commutants. D'après le théorème d'Arnold-Liouville [3] et [18], la variété $\widetilde{\mathbf{F}}$ est un tore complexe et comme celui-ci possède un plongement projectif, alors $\widetilde{\mathbf{F}}$ est une surface abélienne. Par conséquent, on a le:

Théorème 2. La fibre $\mathbf{F}$ (2.3) forme la partie affine d'une surface abélienne $\widetilde{\mathbf{F}}$ et le système (2.2) est algébriquement complètement intégrable.

\section{Surface abélienne en tant que variété de Prym.}

Soit $\left(a_{1}, b_{1}, A, B, a_{2}, b_{2}\right)$ une base de cycles de $\Gamma$ de telle façon que les indices d'intersection de cycles deux à deux s'écrivent: $A o B=1, a_{i} o b_{j}=\delta_{i j}$ (symbole de Kroneker), $a_{i} o a_{j}=a_{i} o A=a_{i} o B=b_{i} o b_{j}=b_{i} o A=b_{i} o B=$ $A o A=B o B=0$ et qu'en outre: $\sigma\left(a_{1}\right)=a_{2}, \sigma\left(b_{1}\right)=b_{2}, \sigma(A)=-A$, $\sigma(B)=-B$ pour l'involution $\sigma$ (2.8). Comme $\Gamma$ est une surface de Riemann hyperelliptique de genre 3 , alors les trois différentielles holomorphes sur $\Gamma$ sont

$$
\omega_{0}=\frac{\alpha d \alpha}{\beta}, \omega_{1}=\frac{\alpha^{2} d \alpha}{\beta}, \omega_{2}=\frac{d \alpha}{\beta},
$$

et évidemment $\sigma^{*}\left(\omega_{0}\right)=\omega_{0}, \sigma^{*}\left(\omega_{k}\right)=-\omega_{k}, k=1,2$. Rappelons que l'involution $\sigma$ échangeant les feuillets du revêtement double $\Gamma \rightarrow \Gamma_{0}$, identifie $\Gamma_{0}$ au quotient $\Gamma / \sigma$. Cette involution induit une involution $\sigma: \operatorname{Jac}(\Gamma) \rightarrow$ $\operatorname{Jac}(\Gamma)$ et modulo un sous-groupe discret, la variété jacobienne $\operatorname{Jac}(\Gamma)$ se décompose en deux parties : une partie paire à savoir $\Gamma_{0}$ et une partie impaire qui n'est autre que la variété de $\operatorname{Prym} \operatorname{Prym}\left(\Gamma / \Gamma_{0}\right)$. Soit

$$
\left(\begin{array}{cccccc}
\omega_{0}(A) & \omega_{0}(B) & \omega_{0}\left(a_{1}\right) & \omega_{0}\left(b_{1}\right) & \omega_{0}\left(a_{2}\right) & \omega_{0}\left(b_{2}\right) \\
\omega_{1}(A) & \omega_{1}(B) & \omega_{1}\left(a_{1}\right) & \omega_{1}\left(b_{1}\right) & \omega_{1}\left(a_{2}\right) & \omega_{1}\left(b_{2}\right) \\
\omega_{2}(A) & \omega_{2}(B) & \omega_{2}\left(a_{1}\right) & \omega_{2}\left(b_{1}\right) & \omega_{2}\left(a_{2}\right) & \omega_{2}\left(b_{2}\right)
\end{array}\right)
$$

la matrice des périodes de $\operatorname{Jac}(\Gamma)$ où $\omega_{k}(*)=\int_{*} \omega_{k}, k=1,2,3$. Or 


$$
\begin{aligned}
\omega_{0}(A) & =\omega_{0}(B)=0, \\
\omega_{0}\left(a_{2}\right) & =\omega_{0}\left(a_{1}\right), \\
\omega_{0}\left(b_{2}\right) & =\omega_{0}\left(b_{1}\right), \\
\omega_{k}\left(a_{2}\right) & =-\omega_{k}\left(a_{1}\right), k=1,2, \\
\omega_{k}\left(b_{2}\right) & =-\omega_{k}\left(b_{1}\right), k=1,2,
\end{aligned}
$$

donc la matrice précédente s'écrit sous la forme

$$
\left(\begin{array}{llllll}
0 & 0 & \omega_{0}\left(a_{1}\right) & \omega_{0}\left(b_{1}\right) & \omega_{0}\left(a_{1}\right) & \omega_{0}\left(b_{1}\right) \\
\omega_{1}(A) & \omega_{1}(B) & \omega_{1}\left(a_{1}\right) & \omega_{1}\left(b_{1}\right) & -\omega_{1}\left(a_{1}\right) & -\omega_{1}\left(b_{1}\right) \\
\omega_{2}(A) & \omega_{2}(B) & \omega_{2}\left(a_{1}\right) & \omega_{2}\left(b_{1}\right) & -\omega_{2}\left(a_{1}\right) & -\omega_{2}\left(b_{1}\right)
\end{array}\right) .
$$

En effectuant des combinaisons linéaires simples sur les colonnes, on obtient les deux matrices suivantes:

$$
\left(\begin{array}{llllll}
0 & 0 & \omega_{0}\left(a_{1}\right) & \omega_{0}\left(b_{1}\right) & 2 \omega_{0}\left(a_{1}\right) & 2 \omega_{0}\left(b_{1}\right) \\
\omega_{1}(A) & \omega_{1}(B) & \omega_{1}\left(a_{1}\right) & \omega_{1}\left(b_{1}\right) & 0 & 0 \\
\omega_{2}(A) & \omega_{2}(B) & \omega_{2}\left(a_{1}\right) & \omega_{2}\left(b_{1}\right) & 0 & 0
\end{array}\right)
$$

et

$$
\left(\begin{array}{llllll}
0 & 0 & \omega_{0}\left(a_{1}\right) & \omega_{0}\left(b_{1}\right) & 0 & 0 \\
\omega_{1}(A) & \omega_{1}(B) & \omega_{1}\left(a_{1}\right) & \omega_{1}\left(b_{1}\right) & 2 \omega_{1}\left(a_{1}\right) & 2 \omega_{1}\left(b_{1}\right) \\
\omega_{2}(A) & \omega_{2}(B) & \omega_{2}\left(a_{1}\right) & \omega_{2}\left(b_{1}\right) & 2 \omega_{2}\left(a_{1}\right) & 2 \omega_{2}\left(b_{1}\right)
\end{array}\right) .
$$

Notons que

$$
\left(2 \omega_{0}\left(a_{1}\right) 2 \omega_{0}\left(b_{1}\right)\right),
$$

est la matrice des périodes de $\Gamma_{0}$, tandis que

$$
\Omega=\left(\begin{array}{llll}
\omega_{1}(A) & \omega_{1}(B) & 2 \omega_{1}\left(a_{1}\right) & 2 \omega_{1}\left(b_{1}\right) \\
\omega_{2}(A) & \omega_{2}(B) & 2 \omega_{2}\left(a_{1}\right) & 2 \omega_{2}\left(b_{1}\right)
\end{array}\right)
$$

est celle de $\operatorname{Prym}\left(\Gamma / \Gamma_{0}\right)$. Considérons l'application (uniformisante)

$$
\widetilde{\mathbf{F}} \rightarrow \mathbb{C}^{2} / L_{\Lambda}: p \mapsto \int_{p_{0}}^{p}\left(\begin{array}{l}
d t_{1} \\
d t_{2}
\end{array}\right),
$$

où $\left(d t_{1}, d t_{2}\right)$ est une base (considérée dans la Section 2$)$ de différentielles holomorphes sur $\widetilde{\mathbf{F}}$ telles que: $\left.d t_{k}\right|_{\Gamma}=\omega_{k}, k=1,2$,

$$
L_{\Lambda}=\left\{\sum_{k=1}^{4} n_{k}\left(\begin{array}{l}
d t_{1} \\
d t_{2}
\end{array}\right)\left(\nu_{k}\right): n_{k} \in \mathbb{Z}\right\},
$$

est le réseau associé à la matrice des périodes

$$
\Lambda=\left(\begin{array}{llll}
d t_{1}\left(\nu_{1}\right) & d t_{1}\left(\nu_{2}\right) & d t_{1}\left(\nu_{3}\right) & d t_{1}\left(\nu_{4}\right) \\
d t_{2}\left(\nu_{1}\right) & d t_{2}\left(\nu_{2}\right) & d t_{2}\left(\nu_{3}\right) & d t_{2}\left(\nu_{4}\right)
\end{array}\right)
$$


et $\left(\nu_{1}, \nu_{2}, \nu_{3}, \nu_{4}\right)$ une base de cycles dans le groupe d'homologie $H_{1}(\widetilde{\mathbf{F}}, \mathbb{Z})$. D'après le théorème de Lefschetz sur les sections hyperplanes [6], l'application $H_{1}(\Gamma, \mathbb{Z}) \rightarrow H_{1}(\widetilde{\mathbf{F}}, \mathbb{Z})$ induite par l'inclusion $\Gamma \hookrightarrow \widetilde{\mathbf{F}}$ est surjective et par conséquent on peut trouver quatre cycles $\nu_{1}, \nu_{2}, \nu_{3}, \nu_{4}$ sur la surface de Riemann $\Gamma$ tels que:

$$
\Lambda=\left(\begin{array}{llll}
\omega_{1}\left(\nu_{1}\right) & \omega_{1}\left(\nu_{2}\right) & \omega_{1}\left(\nu_{3}\right) & \omega_{1}\left(\nu_{4}\right) \\
\omega_{2}\left(\nu_{1}\right) & \omega_{2}\left(\nu_{2}\right) & \omega_{2}\left(\nu_{3}\right) & \omega_{2}\left(\nu_{4}\right)
\end{array}\right)
$$

et

$$
L_{\Lambda}=\left\{\sum_{k=1}^{4} n_{k}\left(\begin{array}{l}
\omega_{1} \\
\omega_{2}
\end{array}\right)\left(\nu_{k}\right): n_{k} \in \mathbb{Z}\right\} .
$$

Ces cycles sont $\nu_{1}=a_{1}, \nu_{2}=b_{1}, \nu_{3}=A, \nu_{4}=B$ et ils engendrent $H_{1}(\widetilde{\mathbf{F}}, \mathbb{Z})$ de telle sorte que

$$
\Lambda=\left(\begin{array}{llll}
\omega_{1}\left(a_{1}\right) & \omega_{1}\left(b_{1}\right) & \omega_{1}(A) & \omega_{1}(B) \\
\omega_{2}\left(a_{1}\right) & \omega_{2}\left(b_{1}\right) & \omega_{2}(A) & \omega_{2}(B)
\end{array}\right)
$$

est une matrice de Riemann. On montre que $\Lambda=\Omega^{\star}$; la matrice des périodes de $\operatorname{Prym}\left(\Gamma / \Gamma_{0}\right)$ duale de $\operatorname{Prym}\left(\Gamma / \Gamma_{0}\right)$. Dès lors, les deux variétés abéliennes $\widetilde{\mathbf{F}}$ et $\operatorname{Prym}\left(\Gamma / \Gamma_{0}\right)$ sont analytiquement isomorphes au même tore complexe $\mathbb{C}^{2} / L_{\Lambda}$ et d'après le théorème de Chow, ces variétés sont algébriquement isomorphes. Par conséquent, on a le:

Théorème 3. La surface abélienne $\widetilde{\mathbf{F}}$ qui complète la fibre $\mathbf{F}$ (2.3) peut être identifiée à la duale d'une variété de Prym $\operatorname{Prym}\left(\Gamma / \Gamma_{0}\right)$ du revêtement double (2.9).

\section{References}

[1] M. Adler and P. van Moerbeke, The complex geometry of the Kowalewski-Painlevé analysis, Invent. Math., 7 (1989), 3-51, MR 90f:58079, Zbl 0678.58020.

[2] _ The Kowalewski and Hénon-Heiles motions as Manakov geodesic flows on $\mathrm{SO}(4)$ - a two-dimensional family of Lax pairs, Comm. Math. Phys., 113 (1987), 659-700, MR 89b:58085, Zbl 0647.58022.

[3] V.I. Arnold, Mathematical Methods in Classical Mechanics, Springer-Verlag, BerlinHeidelberg-New York, 1978, MR 57 \#14033b, Zbl 0386.70001.

[4] T. Bountis, H. Segur and F. Vivaldi, Integrable Hamiltonian systems and the Painlevé property, Phys. Rev. A, 25 (1982), 1257-1264, MR 83j:58056.

[5] L. Gavrilov, Bifurcations of invariant manifolds in the generalized Hénon-Heiles system, Physica D, 34 (1989), 223-239, MR 90h:58040, Zbl 0689.58014.

[6] P.A. Griffiths and J. Harris, Principles of Algebraic Geometry, Wiley-Interscience, New York, 1978, MR 80b:14001, Zbl 0408.14001. 
[7] M. Hénon and C. Heiles, The applicability of the third integral of motion; some numerical experiments, Astron. J., 69 (1964), 73-79, MR 28 \#1969.

[8] S. Kowalevski, Sur le problème de la rotation d'un corps solide autour d'un point fixe, Acta Math., 12 (1989), 177-232, CMP 1916 790, Zbl 21.0935.01.

[9] A. Lesfari, Une approche systématique à la résolution du corps solide de Kowalewski, C. R. Acad. Sci. Paris, 302, série I, 1986, 347-350, MR 87m:58072, Zbl 0606.34012.

[10] _ Abelian surfaces and Kowalewski's top, Ann. Scient. Éc. Norm. Sup., Paris, $4^{e}$ série, 21 (1988), 193-223, MR 89k:58125, Zbl 0667.58019.

[11] _ On affine surface that can be completed by a smooth curve, Result. Math., 35 (1999), 107-118, MR 2000d:14046, Zbl 0947.14022.

[12] _ Geodesic flow on $S O(4)$, Kac-Moody Lie algebra and singularities in the complex t-plane, Publicacions Matemàtiques, Barcelona, 43 (1999), 261-279, MR 2000f:37078, Zbl 0968.35010.

[13] _ Une méthode de compactification de variétés liées aux systèmes dynamiques, Les cahiers de la recherche, Rectorat-Université Hassan II Aïn Chock, Casablanca, Maroc, I, 1 (1999), 147-157.

[14] _ Completely integrable systems: Jacobi's heritage, J. Geom. Phys., 31 (1999), 265-286, MR 2000f:37077, Zbl 0937.37046.

[15] - The problem of the motion of a solid in an ideal fluid. Integration of the Clebsch's case, Nonlinear Diff. Eq. Appl., 8 (2001), 1-13, MR 2002e:70007, Zbl 0982.35085.

[16] _ The generalized Hénon-Heiles system, abelian surfaces and algebraic complete integrability, Reports on Math. Phys., 47 (2001), 9-20, MR 2002b:37093.

[17] _ A new class of integrable systems, Arch. Math., 77 (2001), 347-353, MR 2002e:37091, Zbl 0996.70014.

[18] _ Le théorème d'Arnold-Liouville et ses conséquences, Elem. Math., 58(1) (2003), 6-20, CMP 1961831.

[19] D. Mumford, Tata Lectures on Theta II, Progress in Math., Birkhaüser, Boston, 1984, MR 86b:14017, Zbl 0549.14014.

Received February 24, 2000.

Université Chouaï̈ Doukkali

FACUlté Des SCIENCES

DÉPartement de Mathématiques

B.P. 20, EL-JADIDA

MAROC

E-mail address: lesfari@ucd.ac.ma 